\title{
Health economics agenda in the 21st century - Analysis of research publication trends in health economics from 2000 to 2020
}

\author{
Ozren Polašek ${ }^{1}$ (1) \\ 1 The Croatian Centre for Global Health, University of Split School of Medicine, Split, Croatia \\ Keywords: bibliometrics, economics, trends \\ https://doi.org/10.52872/001c.24316
}

\section{Journal of Global Health Economics and Policy}

Vol. 1, 2021

\begin{abstract}
Background
The aim of this study was to assess the bibliometric output related to health economics from 2000 to 2020 .
\end{abstract}

\section{Methods}

Published papers that were indexed in Pubmed were enumerated and later stratified by regions.

\begin{abstract}
Results
A declining publication trend was observed, with the most articles recorded in 2015, followed by a steady decline ever since. From 2015 to 2020, as many as 42 thousand articles less was published compared to the projected trend of growth recorded from 2000 to 2015. This decline was the strongest for articles related to Europe and the Americas, while articles related to Africa, Asia, and Australia were rising.
\end{abstract}

\section{Conclusions}

A steady decline of health economics research output was observed during the past five years, which was to a degree negated by the rising output from the low- and middle-income countries.

Understanding any research field often begins with a generalized search, where some of the first questions are often related to research publication output. Our fascination with this metric stems from the "publish or perish paradigm", ${ }^{1}$ regardless on the downsides of this approach. ${ }^{2,3}$ This kind of analysis enables not only broad insight, but can further describe trends useful in critical analysis of the research field. ${ }^{4,5}$

Previous analysis of the field of health economics reported very favourable patterns, and suggested likely pathways of development, published in the January of $2016 .^{6}$ However, the situation seems to have changed. During 2000-2020 there were just over a million articles indexed by PubMed (https://pubmed.ncbi.nlm.nih.gov/), which had utilized "health economics" as a keyword (search performed May 14, 2021). Breakdown according to year suggested an average value of over 50,000 articles annually, with the greatest number of all articles published in 2015 (Figure 1, grey bars; Table 1, published). Interestingly, the number of published articles seems to have declined afterwards, never again surpassing the peak in 2015. The situation becomes even clearer when these number are adjusted to the total number of articles indexed by PubMed, which was constantly rising, suggesting that the share of articles related to health economics was on a steady decline (Figure 1, black line). While health economics was mentioned in close to $7 \%$ of all articles in 2000, this percentage had declined to only $3 \%$ in 2020. Although one might argue that 2020 is indeed a special case due to the COVID-19 pandemic, the decline is clearly seen even in the years preceding this. Actually, if we consider the years 2000-2015 as the referent ones, it is possible to calculate the projected number of articles in a linear projection model. Such a model has an $\mathrm{R}^{2}$ of 0.99 , and suggests that the predicted number of published articles during 2016-2020 should be slightly over 333 thousand articles, while only 291 thousand articles were published during this period. This means that the field of health economics had a net loss of over 42 thousand articles, just during these five years (Table 1, column predicted). The year 2020 is even more worrying, since by adding another keyword, COVID seems to deal an additional blow to the filed; the removal of the COVID related articles from the 2020 count (by excluding the papers that also had this keyword), suggested the additional loss of 7600 articles, suggesting that the number of published articles that were not dealing with COVID had declined from slightly over 60 thousand in 2019 to 40 thousand in 2020 , or a decline of $32 \%$.

A more in-depth analysis with the two most common study types: the cost-effectiveness and the cost-benefit analysis, suggests minor deviations from the constant share (Figure 1, lower black lines). At the very least, it seems that the methodologically more demanding types of studies re- 
Table 1. Number of published articles with the selected keywords

\begin{tabular}{|c|c|c|c|c|c|c|c|c|}
\hline \multirow[b]{2}{*}{ Year } & \multirow[b]{2}{*}{$\begin{array}{l}\text { PubMed, all } \\
\text { articles }\end{array}$} & \multicolumn{7}{|c|}{ Key words: "health economics" } \\
\hline & & $\begin{array}{l}\text { Published } \\
\text { articles }\end{array}$ & $\begin{array}{c}\text { Predicted } \\
\text { number* }\end{array}$ & Africa & Asia & Europe & Australia & America \\
\hline 2000 & 532,505 & 35,720 & $35,247.4$ & 884 & 1,620 & 7,622 & 941 & 16,394 \\
\hline 2001 & 547,570 & 38,029 & $36,999.8$ & 909 & 1,850 & 8,797 & 1,123 & 17,269 \\
\hline 2002 & 565,383 & 38,184 & $38,752.2$ & 902 & 1,899 & 8,221 & 1,236 & 17,745 \\
\hline 2003 & 594,535 & 40,543 & $40,504.6$ & 931 & 2,314 & 8,847 & 1,194 & 18,305 \\
\hline 2004 & 639,708 & 42,508 & $42,257.0$ & 1,007 & 2,483 & 8,976 & 1,403 & 19,202 \\
\hline 2005 & 700,230 & 43,855 & $44,009.4$ & 1,091 & 2,617 & 9,236 & 1,540 & 19,180 \\
\hline 2006 & 749,775 & 45,997 & $45,761.8$ & 1,138 & 2,758 & 10,110 & 1,658 & 19,140 \\
\hline 2007 & 786,530 & 46,635 & $47,514.2$ & 1,401 & 3,064 & 10,183 & 1,743 & 19,003 \\
\hline 2008 & 836,935 & 48,543 & $49,266.6$ & 1,363 & 3,296 & 10,438 & 1,824 & 19,028 \\
\hline 2009 & 877,314 & 49,125 & $51,019.0$ & 1,417 & 3,354 & 10,466 & 1,825 & 18,798 \\
\hline 2010 & 941,689 & 52,890 & $52,771.4$ & 1,706 & 3,684 & 11,404 & 2,159 & 20,273 \\
\hline 2011 & $1,019,688$ & 54,557 & $54,523.8$ & 1,795 & 3,877 & 11,634 & 2,326 & 20,035 \\
\hline 2012 & $1,088,565$ & 57,031 & $56,276.2$ & 1,968 & 4,231 & 12,330 & 2,390 & 20,330 \\
\hline 2013 & $1,148,963$ & 59,315 & $58,028.6$ & 2,072 & 4,538 & 12,595 & 2,681 & 20,107 \\
\hline 2014 & $1,203,220$ & 62,644 & $59,781.0$ & 2,571 & 4,774 & 13,437 & 3,306 & 20,844 \\
\hline 2015 & $1,254,691$ & 63,330 & $61,533.4$ & 2,657 & 4,934 & 13,534 & 3,737 & 20,485 \\
\hline 2016 & $1,280,920$ & 61,760 & $63,285.8$ & 2,681 & 4,989 & 12,440 & 3,844 & 19,952 \\
\hline 2017 & $1,297,766$ & 60,446 & $65,038.2$ & 2,751 & 4,996 & 10,777 & 4,106 & 19,373 \\
\hline 2018 & $1,338,297$ & 60,582 & $66,790.6$ & 2,926 & 5,605 & 10,291 & 4,389 & 18,702 \\
\hline 2019 & $1,397,581$ & 60,479 & $68,543.0$ & 3,043 & 6,366 & 9,814 & 4,429 & 17,736 \\
\hline 2020 & $1,617,691$ & 48,495 & $70,295.4$ & 2,700 & 5,428 & 7,036 & 3,384 & 13,051 \\
\hline
\end{tabular}

*Based on the linear trend projection of the 2000-2015, used to predict the number of articles for 2016-2020

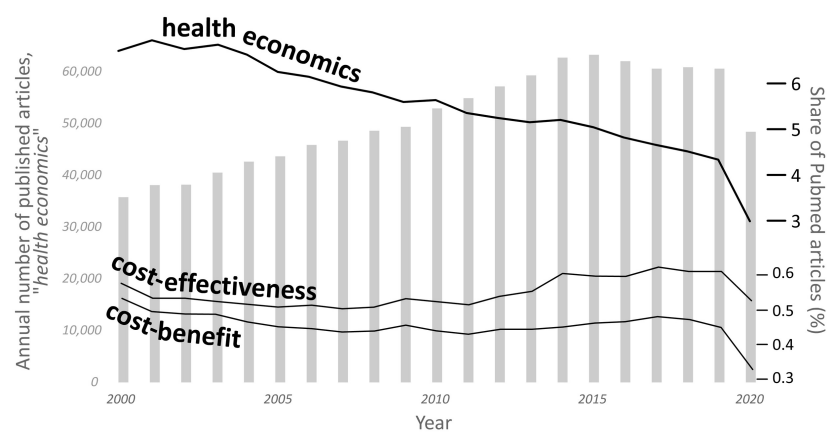

Figure 1. Comparison of the total number of articles with key words "health economics" (grey columns) and the rate of these articles, expressed as the share of total number of PubMed indexed publications in the same year (upper black line)

Lower two black lines denote similar ratios of articles with key words "cross-effectiveness" and "cost-benefit".

tained their constant share in the total number of published articles on PubMed, without an indication of the decline that was obvious for the entire field of health economics.

Although methodologically somewhat unreliable, the addition of the region as yet another keyword suggested that the number of articles related to Europe and America were on a decline, while those related to Asia, Africa and Australia were substantially rising (Table 1).

What could be the reason for this stagnation and decline? Does this mean that we measured it all? Is there nothing more to evaluate? Hardly. If ever, we need health economics now, in the time of global pandemic crises, to assist and aid in decision-making process. What more, we need to substantially expand outreach to health economic studies, the use of evidence-based methods and aim for the best possible evidence. These tasks are very demanding, as they have roots in the improvements of the data collection process, health care organization and health systems, which are all almost daunting tasks on their own. This will become especially important in the post-COVID era, ${ }^{7-9}$ which will require substantial improvements in the way health care is being organized and managed, globally.

\section{FUNDING}

None received.

\section{AUTHOR CONTRIBUTIONS}

OP is the sole author. 


\section{COMPETING INTERESTS}

OP is the Editor-in-Chief of the Journal of Global Health Economics and Policy. To ensure that any possible conflict of interest relevant to the journal has been addressed, this article was reviewed according to best practice guidelines of international editorial organizations. The author has completed the ICMJE Unified Competing Interest form (available upon request from the author) and declares no conflicts of interest.

Submitted: May 16, 2021 CEST, Accepted: May 20, 2021 CEST 


\section{REFERENCES}

1. Publish or perish. Nature. 2010;467(7313):252.

2. Halperin EC. Publish or perish--and bankrupt the medical library while we're at it. Academic medicine: journal of the Association of American Medical Colleges. 1999;74(5):470-472.

3. Oransky I. How Publish or Perish Promotes Inaccuracy in Science-and Journalism. AMA Journal of Ethics. 2015;17(12):1172-1175. doi:10.1001/journal ofethics.2015.17.12.sect1-1512

4. Zheng S, He A, Yu Y, Jiang L, Liang J, Wang P. Research trends and hotspots of health-related quality of life: A bibliometric analysis from 2000 to 2019. Health and quality of life outcomes.

$2021 ; 19(1): 130$.

5. Wu H, Li Y, Tong L, Wang Y, Sun Z. Worldwide research tendency and hotspots on hip fracture: A 20-year bibliometric analysis. Archives of osteoporosis. 2021;16(1):73.
6. Pitt C, Goodman C, Hanson K. Economic Evaluation in Global Perspective: A Bibliometric Analysis of the Recent Literature. Health Econ. 2016;25(Suppl 1):9-28. doi:10.1002/hec.3305

7. Arabi YM, Azoulay E, Al-Dorzi HM, et al. How the COVID-19 pandemic will change the future of critical care. Intensive care medicine. 2021;47(3):282-291.

8. Lee JK, Bullen C, Ben Amor Y, et al. Institutional and behaviour-change interventions to support COVID-19 public health measures: A review by the Lancet Commission Task Force on public health measures to suppress the pandemic. International health. Published online May 11, 2021. doi:10.1093/in thealth/ihab022

9. Citerio G. And once the storm is over... ICM will remain the intensivist's beacon. Intensive Care Med. Published online April 28, 2021:1-5. doi:10.1007/s001 34-021-06402-5 